1990

\title{
Spectrophotometry of several yellow supergiants
}

Robert S. Patterson

Missouri State University

Follow this and additional works at: https://bearworks.missouristate.edu/articles-cnas

\section{Recommended Citation}

Patterson, Robert S. "Spectrophotometry of several yellow supergiants." The Astronomical Journal 99 (1990): 1953-1960.

This article or document was made available through BearWorks, the institutional repository of Missouri State University. The work contained in it may be protected by copyright and require permission of the copyright holder for reuse or redistribution.

For more information, please contact BearWorks@library.missouristate.edu. 


\title{
SPECTROPHOTOMETRY OF SEVERAL YELLOW SUPERGIANTS
}

\author{
ROBERT S. PATTERSON ${ }^{\text {a) }}$ \\ Department of Physics and Astronomy, Southwest Missouri State University, Springfield, Missouri 65804 \\ Received 5 December 1989; revised 26 February 1990
}

\begin{abstract}
Spectrophotometric observations of $20 \mathrm{~F}, \mathrm{G}$, and $\mathrm{K}$ spectral class supergiants and bright giants have been obtained with the Harvard College Observatory scanner on the No. $10.9 \mathrm{~m}$ telescope at Kitt Peak National Observatory. The scans cover the wavelength range 3510-5520 $\AA$ with a resolution of $30 \AA$. The observed flux distributions have been corrected for instrumental effects, atmospheric extinction, and interstellar reddening. The resulting intrinsic flux distributions have been compared to theoretical flux distributions based on the models of Kurucz in order to estimate effective temperatures and surface gravities for eight of these stars.
\end{abstract}

\section{INTRODUCTION}

In order to understand properly the pulsation and evolution of Type I (classical) Cepheid variable stars (spectral classes F-K, luminosity classes I-II), accurate knowledge of the effective temperature $T_{\text {eff }}$ of Cepheids is required. In a review paper, Pel (1985) discussed the current accuracy of Cepheid $T_{\text {eff }}$ determinations. He examined temperature scales produced by Johnson (1966), Böhm-Vitense (1972), Schmidt (1972a,b), van Paradijs (1973), Blackwell and Shallis (1977), Flower (1977), and Bell and Gustafsson (1978) for nonvariable supergiants with spectral classes similar to those of the Cepheids, and by Oke (1961), Kraft (1961), Parsons (1971, 1974), Schmidt (1972a,b), and Pel (1978) for Type I Cepheids. Pel placed the uncertainty in determination of Cepheid $T_{\text {eff }}$ at $\pm 190 \mathrm{~K}$, and pointed out that this uncertainty is large enough to prevent accurate placement of observational limits on the Cepheid instability strip.

One means of determining the effective temperature of a star is to compare its intrinsic flux distribution to the flux distribution predicted by a theoretical stellar model. The intrinsic flux distribution is not directly observable, of course, but can be determined from spectrophotometric observations by correcting them for interstellar extinction, as well as all instrumental effects and atmospheric extinction. This paper presents the results of a comparison of the intrinsic flux distributions of $F, G$, and $K$ spectral class supergiants and bright giants of luminosity classes $\mathrm{Ia}, \mathrm{Ib}$, and $\mathrm{Ib}$-II obtained from spectrophotometry, with theoretical flux distributions based on the models of Kurucz (1979).

\section{OBSERVATIONS AND REDUCTIONS}

Scans of $20 \mathrm{~F}, \mathrm{G}$, and $\mathrm{K}$ supergiants and bright giants were obtained in August 1980 using the Harvard College Observatory (HCO) scanner on the No. $10.9 \mathrm{~m}$ telescope at Kitt Peak National Observatory (KPNO). The HCO scanner was a dual-channel spectrophotometer with matched S-20 multiplier phototube detectors. Dispersion was produced by a 600 lines $/ \mathrm{mm}$ grating blazed for $7500 \AA$ in the first order. Blocking filters were used to exclude spectra other than the second order. A circular entrance aperture with an angular

a) Visiting Astronomer, Kitt Peak National Observatory, National Optical Astronomy Observatories, operated by the Association of Universities for Research in Astronomy, Inc. under contract to the National Science Foundation. diameter of 27.8 arcsec was used. A bandpass of $30 \AA$ was obtained using a $1.829 \mathrm{~mm}$ exit slit. The HCO scanner output was measured by a pulse counting system and recorded using a Varian computer system.

The program stars are listed in Table I according to their HR number from the Bright Star Catalogue (BSC) (Hoffleit 1982). All of the program stars are brighter than $V=7.0$. Suspected variables are indicated by asterisks. The spectral types of the program stars, given in column 3 , are from the BSC.

When the scans were made, equipment problems led to difficulties in recording the data. The primary data recording mechanism failed to record the data on magnetic tape. Most of the data were recorded on a backup printer, except for $30 \%$ of one night's data, which was lost due to the printer being jammed. The printout that survived was used to transcribe the data to computer punch cards, which were then read into a mainframe IBM computer for reduction. Although an attempt was made to reduce the data in this manner, it did not succeed because insufficient observations of flux standard stars remained for use in correcting the observations for instrumental effects. The data remained in limbo until the author obtained the Image Reduction and Analysis Facility (IRAF)* software, to which he had been introduced while on another observing run at KPNO. It was possible to make "pseudo"-IRAF images from the data in hand and process them in the manner that is normally used for the KPNO Intensified Reticon Scanner (IRS) data. Since calibration files exist in the IRAF software for bright standard stars that were observed with the $\mathrm{HCO}$, it was possible to determine sensitivity functions for the observations and thereby calibrate the data.

Integration times for the spectrophotometry were picked to allow at least 10000 counts per channel at the extremes of the wavelength interval scanned for each star. The resulting high $\mathrm{S} / \mathrm{N}$ ratio produced scans of high precision. $\mathrm{Nu}$ Aql was observed on two successive nights, and the scans were processed separately. The ratio spectrum showed that these relative flux distributions were repeated to within $4 \%$ throughout the wavelength interval. Typically, the uncertainties in the spectrophotometry were about 2-3\%.

A typical example of $\mathrm{HCO}$ data ( $30 \AA$ resolution) reduced by IRAF is shown in Fig. 1 for 35 Cyg (as a bold line),

\footnotetext{
*IRAF is distributed by the National Optical Astronomy Observatories, which is operated by the Association of Universities for Research in Astronomy, Inc., under contract to the National Science Foundation.
} 
TABLE I. Program stars

\begin{tabular}{|c|c|c|c|c|c|c|}
\hline \multirow{2}{*}{$\begin{array}{r}\text { HR } \\
382\end{array}$} & Name & \multicolumn{2}{|c|}{$\begin{array}{c}\text { Spectral } \\
\text { Type }\end{array}$} & \multirow{2}{*}{$\frac{B-V}{0.68}$} & \multirow{2}{*}{$\frac{(B-V)_{0}}{0.17}$} & \multirow{2}{*}{$\frac{E_{B-V}}{0.51}$} \\
\hline & $\phi$ Cas & Fo & Ia & & & \\
\hline 6978 & 45 Dra & F7 & Ib & 0.61 & 0.51 & 0.10 \\
\hline 7008 & $\ldots$ & F8 & $I b-I I$ & 0.78 & 0.58 & 0.20 \\
\hline 7314 * & $\theta$ Lyr & KO & II & 1.26 & 1.05 & 0.21 \\
\hline 7387 * & $\nu \mathrm{Aql}$ & F2 & Ib & 0.60 & 0.24 & 0.36 \\
\hline 7542 & $\ldots$ & F8 & Ib-II & 0.96 & 0.58 & 0.38 \\
\hline 7747 & $\alpha^{\prime}$ Cap & G3 & $\mathrm{Ib}$ & 1.07 & 0.90 & 0.17 \\
\hline $7770 *$ & 35 cyg & F5 & Ib & 0.65 & 0.37 & 0.28 \\
\hline 7834 * & 41 cyg & F5 & II & 0.40 & 0.37 & 0.03 \\
\hline 7892 & $\theta$ Dél & K3 & Ib & 1.53 & 1.31 & 0.22 \\
\hline 8126 & $\ldots$ & G6 & Ib-IIa & 1.09 & 0.96 & 0.13 \\
\hline $8248 *$ & $\ldots$ & K1 & Ibv & 1.81 & 1.24 & 0.57 \\
\hline $8313 *$ & 9 Peg & G5 & Ib & 1.17 & 0.99 & 0.18 \\
\hline 8321 & 12 Peg & Ko & Ib & 1.41 & 1.19 & 0.22 \\
\hline 8374 & $\ldots$ & G8 & Ib & 1.60 & 1.09 & 0.51 \\
\hline 8692 & $\ldots$ & G4 & Ib & 1.14 & 0.94 & 0.20 \\
\hline $8752 *$ & $\cdots$ & G4 & vo & 1.42 & 0.94 & 0.48 \\
\hline 8952 & $\cdots$ & Ko & Ib & 1.80 & 1.19 & 0.61 \\
\hline 8982 * & 104 Aqr & GO & Ib-II & 0.82 & 0.73 & 0.09 \\
\hline 9053 & $\ldots$ & G8 & Ib & 1.16 & 1.09 & 0.07 \\
\hline
\end{tabular}

* Suspected variable

superposed with an IRAF-processed IRS scan of the same star ( 9 Å resolution) obtained by the author in 1987 (Patterson 1990). The close match between these two observed flux distributions confirms the validity of the HCO data.

The observed scans were corrected for interstellar reddening by applying the general reddening law of Schild (1977). The $E_{B-V}$ color excesses used in the correction process are listed in the last column of Table I for each program star. The color excesses are the differences between the values in columns 4 and 5 . The observed $(B-V)$ color indices used for the program stars are given in column 4 , and are the values tabulated in the BSC. The intrinsic $(B-V)_{0}$ color indices used are listed in column 5 , and are the values determined from the literature by Patterson and Neff (1979). These intrinsic color indices are arithmetic averages of the values for luminosity classes I and II supergiants and bright giants reported in the following five sources: Feinstein (1959), Kraft and Hiltner (1961), FitzGerald (1970), Par-

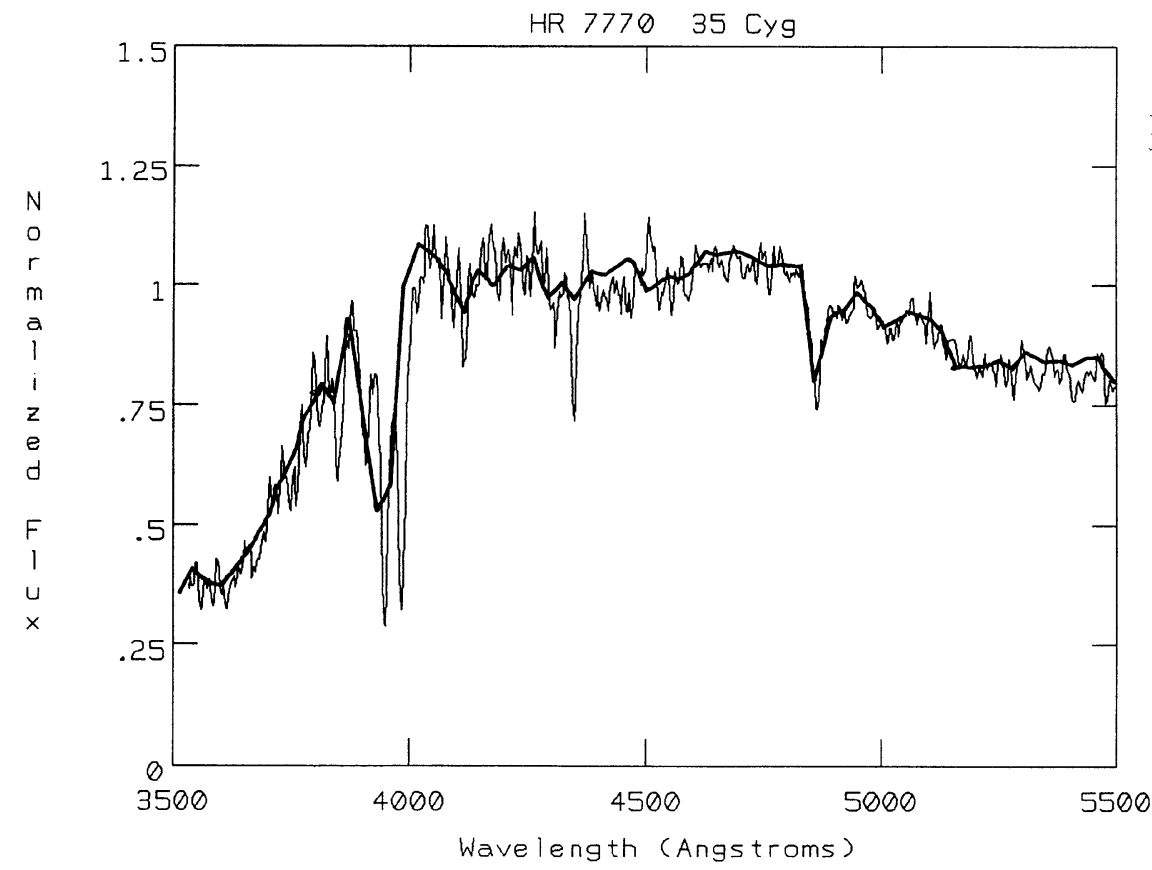

FIG. 1. Comparison of observations of 35 Cyg made with the Harvard scanner (30 $\AA$ resolution) (bold line) and the Intensified Reticon Scanner ( 9 Å resolution) 
sons (1971), and Kron (1978). Over the spectral range F0G5, the standard deviation of the mean intrinsic color indices from these five sources is typically $0.04 \mathrm{mag}$. This contribution to the error in determination of the $T_{\text {eff }}$ of the program stars is in agreement with the estimate of Pel (1985). The adopted intrinsic color indices used here are also in good agreement with the intrinsic color indices found by Grieve and Madore (1986) for Magellanic Cloud luminosity class Ia supergiants of spectral classes F0-G2.

The resulting intrinsic flux distributions were then normalized to the value at $4700 \AA$ for subsequent comparison to the theoretical distributions. These intrinsic flux distributions for all the program stars are presented in Fig. 2.

\section{MODEL ATMOSPHERES}

A subset of the grid of model atmospheres calculated by Kurucz (1979) was selected, with $T_{\text {eff }}$ values ranging from $7500 \mathrm{~K}$ down to the lowest temperature in the grid, $5500 \mathrm{~K}$, by steps of $500 \mathrm{~K}$, and for $\log$ surface gravity $(\log g$ ) values (in cgs units) from 1.5 to 3.0 by steps of 0.5 , all for solar abundance. The Kurucz grid is for LTE model atmospheres computed using a statistical distribution-function representation of the opacity of almost $10^{6}$ atomic lines. The Kurucz fluxes were interpolated to the same resolution as the observations and normalized to the value at $4700 \AA$. For example, Fig. 3 displays the models for the five effective temperatures at $\log g=1.5$.

\section{ANALYSIS}

The intrinsic flux distributions were compared to the theoretical flux distributions using two techniques. The first method used ratio spectra, where the model flux distributions were divided into the intrinsic flux distributions on a point-by-point basis. A good fit is therefore represented by a flat ratio spectrum with values near unity across the wavelength range. Of the 20 program stars, only eight showed flat ratio spectra. These are shown in Fig. 4. The second method was a visual comparison of an intrinsic flux distribution superposed with each member of the theoretical flux distribution grid. The eight program stars that closely matched a theoretical flux distribution are given in Fig. 5, with the bestfit theoretical model superposed in bold. These are the same
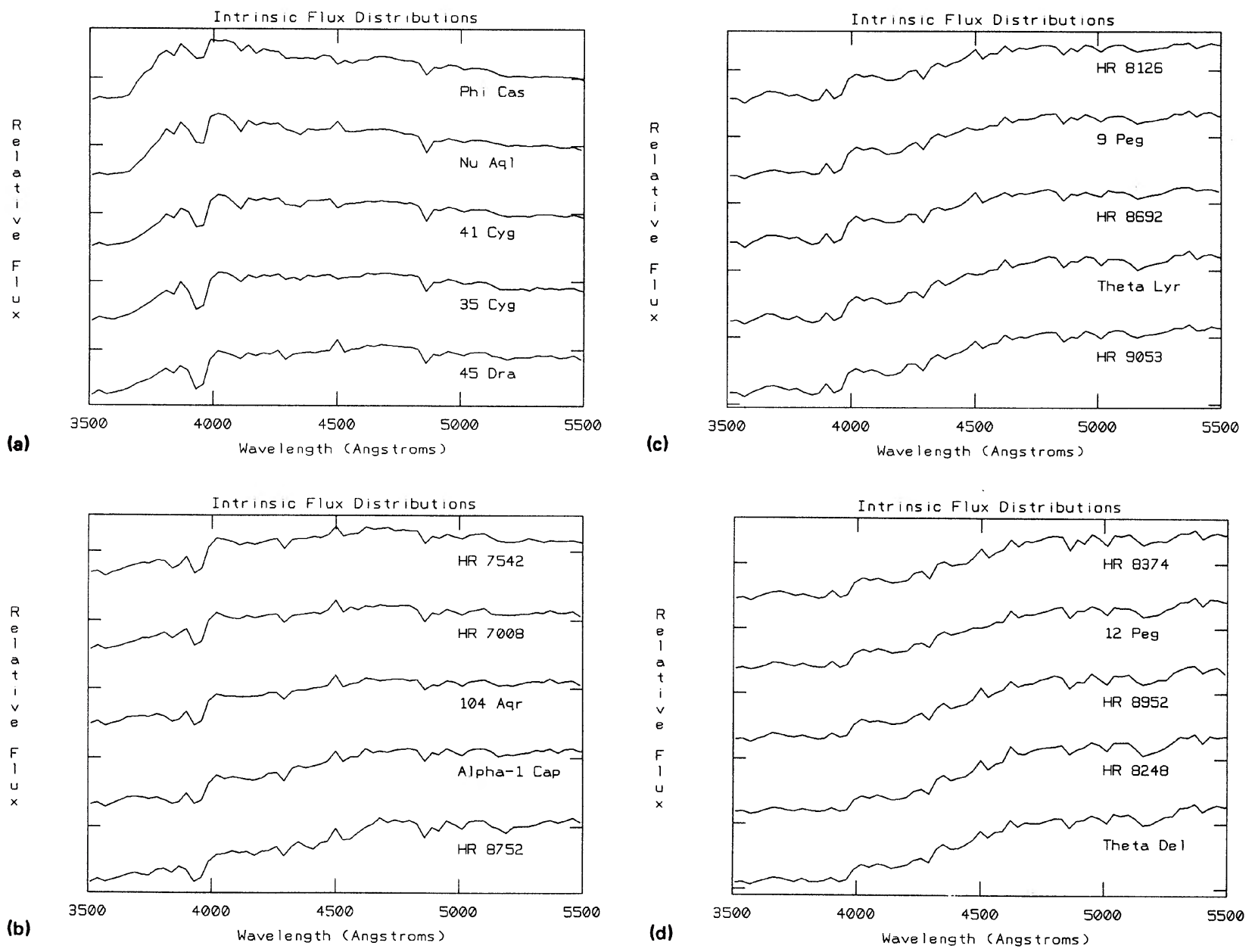

Fig. 2. Intrinsic (dereddened) flux distributions of (a) HR 0382 (Phi Cas), HR 7387 (Nu Aql), HR 7834 (41 Cyg), HR 7770 ( 35 Cyg), and HR 6978 (45 Dra); (b) HR 7542, HR 7008, HR 8982 (104 Aqr), HR 7747 (Alpha-1 Cap), and HR 8752; (c) HR 8126, HR 8313 (9 Peg), HR 8692, HR 7314 (Theta Lyr), and HR 9053; and (d) HR 8374, HR 8321 (12 Peg), HR 8952, HR 8248, and HR 7892 (Theta Del). All distributions are normalized to the value at $4700 \AA$. 


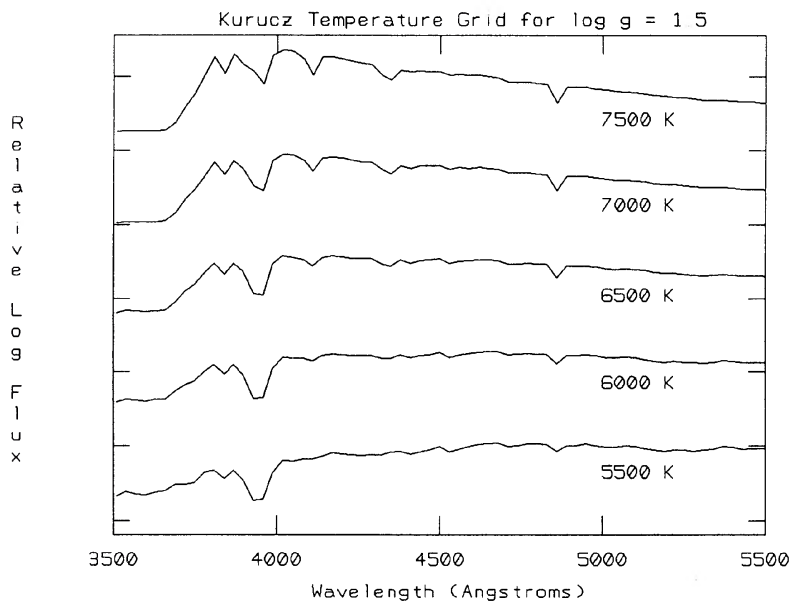

FIG. 3. Theoretical flux distributions based on the Kurucz models for $7500,7000,6500,6000$, and $5500 \mathrm{~K}$, all for $\log g=1.5$, normalized to the value at $4700 \AA$.

eight stars that showed flat ratio spectra. Also shown in Fig. 5 are the intrinsic flux distributions for two stars that are cooler than the low-temperature $(5500 \mathrm{~K})$ cutoff of the Kurucz grid: HR 7747 (Alpha-1 Cap) and HR 8752. The results of the two methods were then averaged to determine the $T_{\text {eff }}$ and log surface gravity for a program star. The ratio spectra and visual overlays indicated that 12 of the program stars have temperatures well below $5500 \mathrm{~K}$. Effective temperatures and log surface gravities determined for the remaining eight program stars are given in Table II.

The observed flux distributions, as determined from the spectrophotometry, have uncertainties of as much as $4 \%$. The published values of observed color index in the BSC are the results of photoelectric measurements and have uncer- tainties of approximately $1 \%$. Contributions to the uncertainties in determinations of temperature and gravity from the intrinsic color indices are a significant source of error and are estimated to be about $5 \%$, as described in Sec. II. The effect of a $5 \%$ uncertainty in the color excess values was investigated by reprocessing the data for $\mathrm{Nu}$ Aql using $E_{B-V}$ values that were 0.05 mag greater and lower than the value adopted in Table I. This led to effective temperatures that were $125 \mathrm{~K}$ higher and lower than the results quoted in Table II. This uncertainty was about the same as the ability to fit an intrinsic flux distribution to the grid using the methods previously described.

The combined uncertainties in the spectrophotometry, the color excesses, and the model fitting amount to about $\pm 250 \mathrm{~K}$ and $\pm 0.25 \log g$ in most cases, except for those stars that were near the lower-temperature cutoff of the grid, in which case the uncertainties quoted are $\pm 375 \mathrm{~K}$ and $\pm 0.5 \log g$.

The temperature scale obtained in this paper is compared to those of Bell and Parsons (1974), Blackwell and Shallis (1977), Böhm-Vitense (1981), Flower (1977), Johnson (1966), Kraft (1961), and Schmidt (1972a,b) in Table III, which lists the zero-point and slope values for linear leastsquares fits to the $\log T_{\mathrm{eff}}-(B-V)_{0}$ relation for the data of each of these investigators. All values in Table III have been recomputed from the original data in order to coincide with the range in $(B-V)_{0}$ studied here, i.e., from about 0.2 to 0.75. The Blackwell and Shallis values in Table III are listed in parentheses because they were determined from only two data points.

There is general agreement among most workers for the zero-point value of the temperature scale, which averages about 3.90. However, the slopes of the temperature scales of the various workers indicated in Table III range from -0.104 to -0.299 . The results of this paper show one of the steepest slopes.

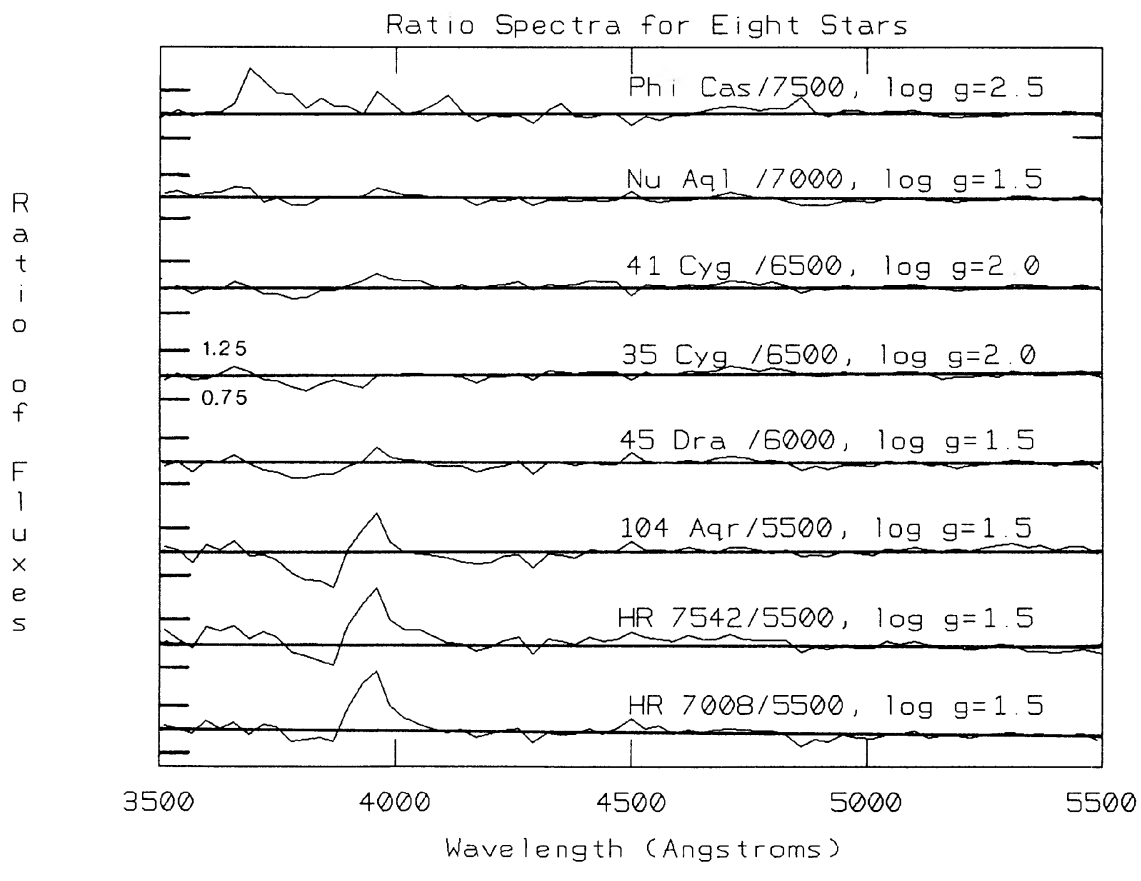

FIG. 4. Ratio spectra for eight stars. These are the intrinsic flux distributions divided by the indicated Kurucz models. The horizontal lines represent 1.0 Marks for ratios of 1.25 and 0.75 are indicated for each spectrum. 


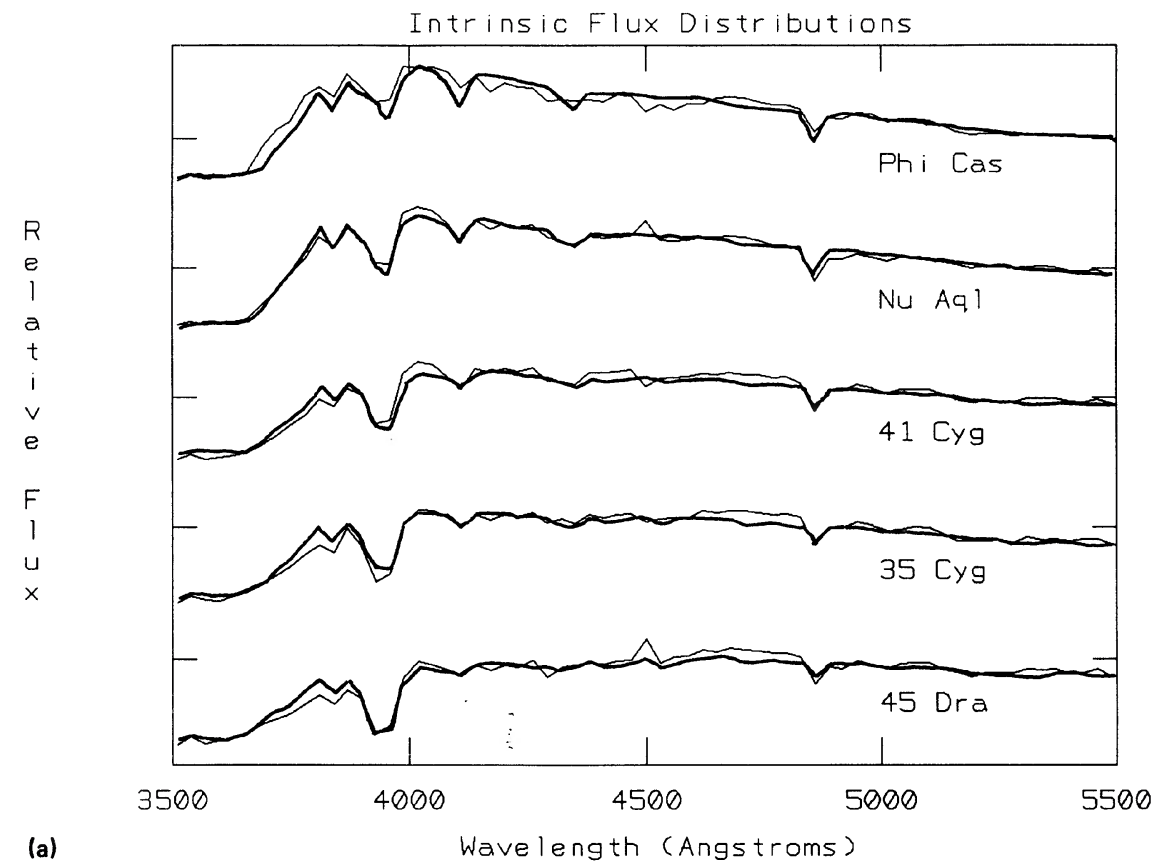

FIG. 5. (a) Normalized intrinsic flux distributions for HR 0382 (Phi Cas), HR 7387 (Nu Aql), HR 7834 (41 Cyg), HR 7770 (35 Cyg), and HR 6978 (45 Dra), each superposed with the best-fit theoretical flux distribution from the Kurucz models (bold lines). (b) Normalized intrinsic flux distributions for HR 7542, HR 7008, HR 8982 (104 Aqr), HR 7747 (Alpha-1 Cap), and HR 8752 , each superposed with the best-fit theoretical flux distribution from the Kurucz models (bold lines). Alpha-1 Cap and HR 8752 are cooler than the lowest temperature $(5500 \mathrm{~K})$ in the Kurucz grid.

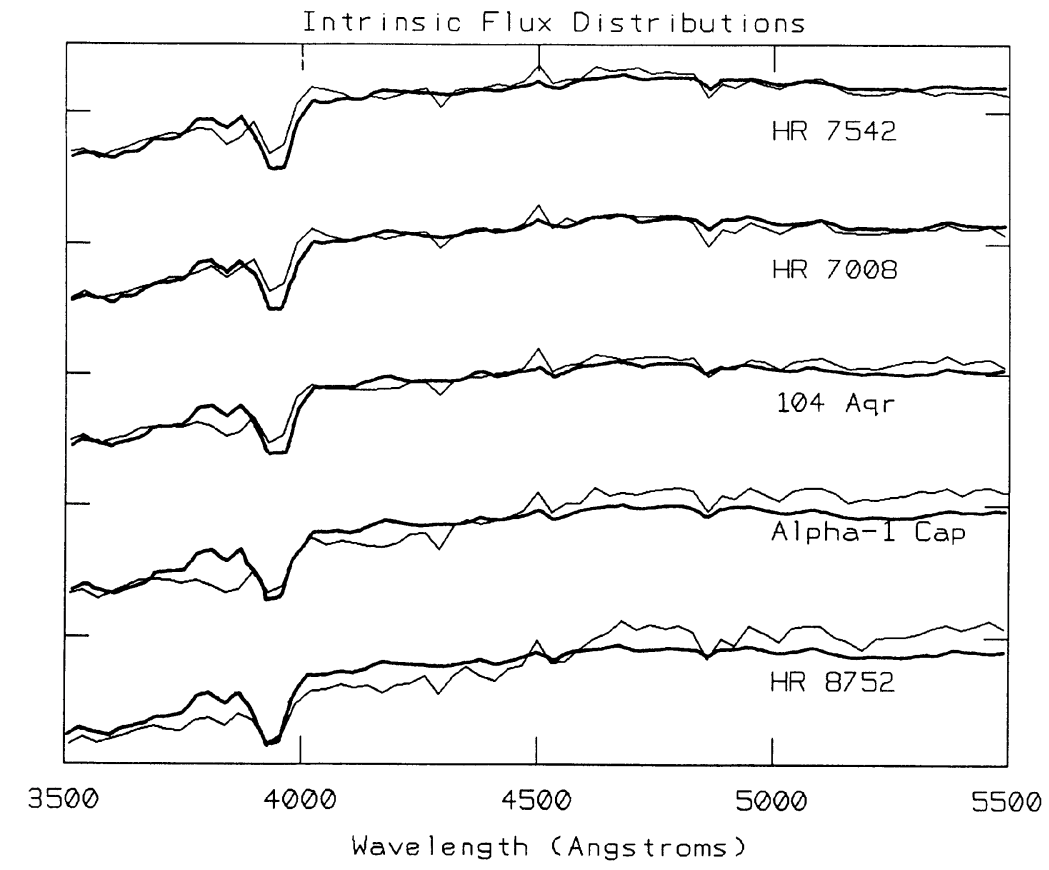

\section{DISCUSSION}

For comparison, the temperature determinations made in this paper are shown in Fig. 6 along with the $T_{\text {eff }}$ data plotted as a function of $(B-V)_{0}$ from several sources: Bell and Parsons (1974), Böhm-Vitense (1981), Flower (1977), Johnson (1966), and Kraft (1961). In preparing Fig. 6, it was necessary to use the original data from the sources listed. It became clear that, in some cases, the $\log T_{\text {eff }}$ data appear linear in $(B-V)_{0}$, while in others they do not. Nonlinear temperature scales include those of Böhm-Vitense (1981),
Flower (1977), and Johnson (1966). Although it might be valid to consider the temperature scale linear over small ranges in $(B-V)_{0}$, as has been done in Table III, it is probably not appropriate over a large range.

Aside from considerations of the nonlinearity of the temperature scale for Cepheids and yellow supergiants is the problem posed by the wide range in slopes obtained by various workers. In particular, for the steeper (more negative) slopes, the use of intrinsic colors to estimate the $T_{\text {eff }}$ of Cepheids from the $\log T_{\text {eff }}-(B-V)_{0}$ relation leads to Baade- 
TABLE II. Effective temperatures and $\log$ surface gravities.

\begin{tabular}{|c|c|c|c|c|c|c|}
\hline \multirow{2}{*}{$\begin{array}{r}\text { HR } \\
382\end{array}$} & Name & \multicolumn{2}{|c|}{$\begin{array}{c}\text { Spectral } \\
\text { Type }\end{array}$} & $T_{\text {eff }}(\mathrm{K})$ & $\log g$ & (c.g.s.) \\
\hline & $\phi$ Cas & Fo & Ia & 7625 & & 2.5 \\
\hline 6978 & 45 Dra & F7 & Ib & 5875 & & 1.0 \\
\hline 7008 & $\ldots$ & F8 & $I b-I I$ & 5500 & & 1.75 \\
\hline 7387 * & $\nu A q l$ & F2 & Ib & 7000 & & 1.625 \\
\hline 7542 & $\cdots$ & F8 & $I b-I I$ & 5500 & & 1.50 \\
\hline 7770* & 35 Cyg & F5 & Ib & 6375 & & 1.875 \\
\hline 7834 * & 41 Cyg & F5 & II & 6500 & & 1.875 \\
\hline 8982 * & $104 \mathrm{Aqr}$ & GO & Ib-II & 5250 & & 1.25 \\
\hline
\end{tabular}

Wesselink radii that are much too small (Böhm-Vitense et al. 1989).

Possible explanations for the steep slope of the temperature scale determined in the present study can be found by examination of the program stars themselves, the models used, and the process used to compare them. Only eight of the program stars had temperatures high enough to compare to the Kurucz grid of models. In order to increase the statistical significance of this method of temperature determination, more stars should be observed. Spectrophotometry of an additional 40 yellow supergiants and bright giants has been obtained, and will be reported on in Patterson (1990). Four of the stars whose temperatures and gravities were determined are listed in the BSC as being suspected of light variation: $\mathrm{Nu} \mathrm{Aql,} 35 \mathrm{Cyg}, 41 \mathrm{Cyg}$, and $104 \mathrm{Aqr}$. None are confirmed variables, and since they are all bright stars, any light variation would be of low amplitude. Therefore, variability of the program stars is ruled out as a source of error for the method used here. According to the BSC, four of the stars are members of multiple systems: Phi Cas, Nu Aql, 35 Cyg, and 104 Aqr. Phi Cas has four companions. The B component is separated by 49 arcsec and is a $V=12 \mathrm{mag}$ star. Component $\mathrm{C}$ is farther (134 arcsec), but is brighter $(V=7.8)$. The entrance slit used on the HCO scanner was 27.8 arcsec in diameter, so that unwanted light from these companions to Phi Cas should not have contaminated the observed flux distributions. However, the $T_{\text {eff }}$ determined here for Phi Cas is considerably higher than the $6700 \mathrm{~K}$ found by Schmidt (1972b), who also fit continuum scans to model distributions. The $T_{\text {eff }}$ found here is $425 \mathrm{~K}$ higher than the $7200 \mathrm{~K}$ found for Phi Cas by Rosenzweig and Anderson (1988) based on comparison of the energy distribution and line profiles to their own spherical, non-LTE, blanketed model of Phi Cas. Lowering the $T_{\text {eff }}$ of Phi Cas by 425 $\mathrm{K}$ would certainly lead to a shallower slope in the present scale. More surprising is the discordance in $\log g=0.4$ found by Rosenzweig and Anderson compared to the 2.5 found here. The Balmer jump seen in Fig. 5(a) for Phi Cas is clearly much too small to correspond to $\log g=0.4$. However, the intrinsic flux distribution for Phi Cas does show an excess compared to the model for wavelengths less than about $4000 \AA$. Sufficient doubt about the result for Phi Cas exists to warrant its omission from the determination of the temperature scale. A recalculation of the slope of the $\log T_{\text {eff }}-(B-V)_{0}$ relation with Phi Cas omitted results in a value of -0.286 , which is only a slight improvement. $\mathrm{Nu}$ Aql and 104 Aqr are also visual binaries whose B components are at 201 and 120 arcsec, respectively. These separations make it unlikely that any contamination of the observed flux distributions occurred. $35 \mathrm{Cyg}$ is, in fact, a

TABLE III. Comparison of temperature scales for $0.2<\left(B-V_{0}<0.75\right.$.

\begin{tabular}{lcc}
\hline \multicolumn{1}{c}{ Reference } & Zero-point & Slope \\
\hline Bell and Parsons (1974) & 3.884 & -0.195 \\
Blackwell and Shallis (1977) & $(3.894$ & $-0.212)$ \\
Böhm-Vitense (1981) & 3.916 & -0.264 \\
Flower (1977) & 3.947 & -0.299 \\
Johnson (1966) & 3.914 & -0.220 \\
Kraft (1961) & 3.887 & -0.176 \\
Schmidt (1972a,b) ${ }^{2}$ & 3.853 & -0.104 \\
Schmidt (1972a,b) & 3.877 & -0.195 \\
& & -0.297 \\
This paper & 3.920 & \\
\hline \hline & & \\
1) Luminosity Class I supergiants &
\end{tabular}


COMPARISON OF TEMPERATURE SCALES

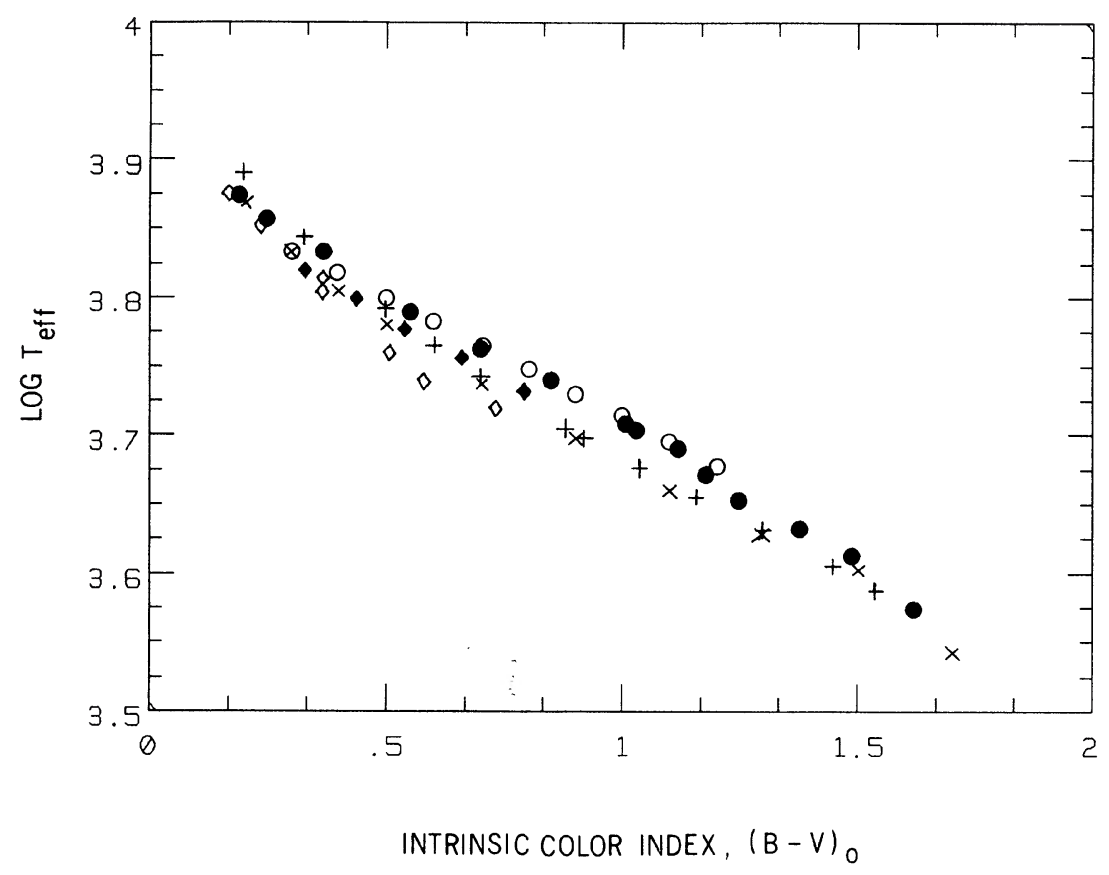

FIG. 6. Comparison of the temperature scale determined by this study with those from five other sources. The data are shown as filled diamonds for Bell and Parsons (1974), crosses for Böhm-Vitense (1981), plus signs for Flower (1977), filled circles for Johnson (1966), open circles for Kraft (1961), and open diamonds for the present study. spectroscopic binary of period 2440 days, and should probably be omitted from consideration here on that basis. This would not, however, reduce the steepness of the $\log T_{\text {eff }}-$ $(B-V)_{0}$ relation, since $41 \mathrm{Cyg}$ has similar values of $T_{\mathrm{eff}}$ and $(B-V)_{0}$. In summary, it does not seem likely that the choice of program stars, other than the small number selected, contributed to the steep slope found here.

The Kurucz models, as utilized here, have two major drawbacks associated with them. First, the models used did not account for non-LTE conditions, molecular opacity, or convection, all of which are important in accurately modeling the cooler stars studied here (Kurucz 1979). This may have led to systematically low determinations of $T_{\text {eff }}$ for the later-type supergiants studied here. Second, only the Kurucz models for solar abundance were used in this study, although other abundances were available in the Kurucz grid. The effect of abundance on the $T_{\text {eff }}$ determination is currently being investigated (Patterson 1990).

If the process used here to determine $T_{\text {eff }}$ and $\log g$ has contributed to a too-steep slope, it could possibly be produced by the spectrophotometric observations, including the equipment used to obtain them, the color excesses adopted, or the interstellar reddening law used to correct the flux distributions. One problem with the spectrophotometry was the HCO instrument itself. Although the precision of the measurements was high, the HCO did not measure flux at all wavelengths simultaneously, as does the IRS, which is certainly an improved scanner, and one less subject to systematic errors. The color excesses used here to deredden the flux distributions were based upon the intrinsic $(B-V)_{0}$ color indices found in the literature. An uncertainty of $5 \%$ in the $E_{B-V}$ color excess values adopted here could, in the worst case, and with Phi Cas excluded, decrease the $T_{\text {eff }}$ at $(B-V)_{0}=0.2$ by $125 \mathrm{~K}$, and increase the $T_{\text {eff }}$ at $(B-V)_{0}$
$=0.7$ by the same amount, and simultaneously lower the minimum value of $(B-V)_{0}$ by 0.05 mag and raise the maximum value of $(B-V)_{0}$ by the same. This would result in a slope of -0.195 , which is actually slightly shallower than the average of the eight values given in Table III. But note that Luck (1982a,b) found $T_{\text {eff }}=5500 \mathrm{~K}, \log g=1.80$ for $104 \mathrm{Aqr}$ and $T_{\text {eff }}=5500 \mathrm{~K}, \log g=2.10$ for $\mathrm{HR} 7008$, in good agreement with the temperatures found here. This agreement is an argument against the need to increase the temperatures of the cooler stars. Finally, the general reddening law used here to correct the scans for interstellar extinction may be insufficient due to small-scale variations in the structure of the interstellar dust distribution.

Future work will therefore concentrate on a much larger sample of similar stars observed with the KPNO IRS in 1987 and 1988. These observations will be compared to a wider subset of improved, convective Kurucz models, including compositions other than solar. In addition, particular attention will be focused on determining more accurate $E_{B-V}$ values for use in the dereddening process, because of the strong effect it has on the results. Finally, reddening laws will be used that are specific to the galactic coordinates of each program star.

I wish to thank KPNO for granting observing time, Don Hayes for his assistance during my observing run, Tony Hayes for helping with the reduction of the data, Julie Pearce and Leah Robie for help in the production of the manuscript, and Jeannette Barnes and NOAO for providing me a copy of IRAF. I would also like to thank an anonymous referee for constructive criticism of the manuscript. I appreciate the support of the Southwest Missouri State University Faculty Research Committee for supplying the funds needed to make the observing run at KPNO in 1980. 


\section{REFERENCES}

Bell, R. A., and Gustafsson, B. (1978). Astron. Astrophys. Suppl. 34, 229. Bell, R. A., and Parsons, S. B. (1974). Mon. Not. R. Astron. Soc. 169, 71 Blackwell, D. E., and Shallis, M. J. (1977). Mon. Not. R. Astron. Soc. 180, 177.

Böhm-Vitense, E. (1972). Astron. Astrophys. 17, 335.

Böhm-Vitense, E. (1981). Annu. Rev. Astron. Astrophys. 19, 295.

Böhm-Vitense, E., Garnavich, P., Lawler M., Mena-Worth, J., Morgan, S., Peterson, E., and Temple, S. (1989). Astrophys. J. 343, 343.

Feinstein, A. (1959). Z. Astrophys. 47, 224.

FitzGerald, M. P. (1970). Astron. Astrophys. 4, 234.

Flower, P. J. (1977). Astron. Astrophys. 54, 31.

Grieve, G. R., and Madore, B.F. (1986). Astrophys. J. Suppl. 62, 427.

Hoffleit, D. (1982). The Bright Star Catalogue, 4th revised ed. (Yale University Observatory, New Haven).

Johnson, H. L. (1966). Annu. Rev. Astron. Astrophys. 4, 193.

Kraft, R. P. (1961). Astrophys. J. 134, 616.

Kraft, R. P., and Hiltner, W. A. (1961). Astrophys. J. 134, 616.

Kron, G. E. (1978). Private communication.

Kurucz, R. L. (1979). Astrophys. J. Suppl. 40, 1.
Luck, R. E. (1982a). Astrophys. J. 256, 177.

Luck, R. E. (1982b). Astrophys. J. 263, 215.

Oke, J. B. (1961). Astrophys. J. 134, 214.

Parsons, S. B. (1971). Mon. Not. R. Astron. Soc. 152, 121.

Parsons, S. B. (1974). In Stellar Stability and Evolution, IAU Symposium No. 59, edited by P. Ledoux, A. Noels, and A. W. Rodgers (Reidel, Dordrecht), p. 55.

Patterson, R. S. (1990). In preparation.

Patterson, R. S., and Neff, J. S. (1979). Astrophys. J. Suppl. 41, 215.

Pel, J. W. (1978). Astron. Astrophys. 62, 75.

Pel, J. W. (1985). In Cepheids: Theory and Observations, IAU Colloquium No. 82, edited by B. F. Madore (Cambridge University, Cambridge), p. 5.

Rosenzweig, P., and Anderson, L. S. (1988). Bull. Am. Astron. Soc. 20, 698.

Schild, R. E. (1977). Astron. J. 82, 337.

Schmidt, E. G. (1972a). Astrophys. J. 174, 595.

Schmidt, E. G. (1972b). Astrophys. J. 174, 605.

van Paradijs, J. (1973). Astron. Astrophys. 23, 369. 